\section{Tabagismo no domicílio e baixa estatura em menores de cinco anos}

\author{
Household smoking and stunting \\ for children under five years
}

\author{
1 Faculdade de Nutrição, \\ Universidade Federal de \\ Mato Grosso, Cuiabá, Brasil. \\ 2 Instituto de Medicina \\ Social, Universidade \\ do Estado do Rio de Janeiro, \\ Rio de Janeiro, Brasil. \\ 3 Escola Nacional de Saúde \\ Pública, Fundação Oswaldo \\ Cruz, Rio de Janeiro, Brasil. \\ Correspondência \\ R. M. V. Gonçalves-Silva \\ Faculdade de Nutrição, \\ Universidade Federal \\ de Mato Grosso. \\ Rua 27, casa 207, Cuiabá, MT \\ 78068-590, Brasil. \\ reveras@uol.com.br
}

\begin{abstract}
Maternal tobacco use during pregnancy is one of the most important factors for low birth weight and length. However, postnatal exposure to environmental tobacco smoke has not been explored in children's growth studies. It is known that smoking is more prevalent in low socioeconomic groups and that stunting is also associated with socioeconomic variables. The objective of this study was to verify the effect of socioeconomic variables and environmental tobacco smoke on growth. Children under five years $(n=2,037)$ attending a primary health care clinic had their weight and stature measured, and a questionnaire on exposure to household passive smoking and socio-demographic characteristics was applied to parents. Prevalence of stunting (defined as a $z$-score lower than -2) was 4.3\%. There was a negative association between stature and smoking during pregnancy, and positive associations with socioeconomic levels, family income, and parents' education. Linear hierarchical regression analysis showed that maternal and paternal smoking remains associated with low stature even after adjusting for maternal smoking during pregnancy and for socio-demographic variables.
\end{abstract}

Child; Body Height; Tobacco Smoke Pollution
Regina M. V. Gonçalves-Silva 1

Joaquim G. Valente ${ }^{2,3}$

Márcia G. F. Lemos-Santos 1

Rosely Sichieri 2

\section{Introdução}

Muitos estudos têm investigado a relação entre os fatores sociais, econômicos, biológicos e a estatura de crianças 1 . Variáveis relacionadas aos antecedentes reprodutivos da mãe e do meio ambiente têm sido freqüentemente associados à estatura das crianças 2 . Dentre os fatores do meio ambiente mais estudados, estão aqueles ligados às condições nutricionais, os quais, por sua vez, sofrem influência das variáveis sócio-econômicas, como renda familiar, escolaridade dos pais, condições de moradia e saneamento, além de presença de doenças infecciosas ou parasitárias, cuidados de higiene e assistência à saúde 3,4.

Uma variável relacionada à condição sócioeconômica das famílias e que, geralmente, não é incluída nos estudos de avaliação nutricional é o tabagismo dos pais e moradores do domicílio, embora o tabagismo durante o período gestacional associe-se ao baixo peso, redução do perímetro cefálico e torácico e ao comprimento de recém-nascidos 5,6,7. No entanto, a conseqüência da exposição ao tabagismo no período pós-natal, para a estatura infantil, não está bem esclarecida. Alguns autores alegam que a menor estatura dos filhos de fumantes em relação à dos não fumantes é decorrente de efeito residual do fumo durante a gravidez 8. Outros pesquisadores referem que a exposição ao tabagismo dos pais pode ser mais importante do que a exposição no útero ${ }^{9}$. 
Pesquisa recente mostrou que o tabagismo é fortemente influenciado pelas variáveis sócio-econômicas, sendo maior a prevalência naqueles domicílios com menor renda familiar, em que os pais têm baixa escolaridade e naqueles em que os moradores exercem ocupação menos qualificada 10.

O objetivo deste estudo foi investigar o efeito da exposição ao tabagismo domiciliar sobre a estatura das crianças menores de cinco anos, após o ajuste para variáveis sócio-econômicas e demográficas, tidas como importantes fatores de confusão.

\section{Métodos}

A população de estudo foram as crianças com idade inferior a sessenta meses que freqüentaram os postos de saúde da cidade de Cuiabá, Mato Grosso, Brasil, para vacinação, no período compreendido entre agosto de 1999 e janeiro de 2000 . A cobertura vacinal da cidade no período foi de $97,0 \%$ para tríplice bacteriana em menores de um ano, e as crianças eram encaminhadas pelo hospital em que nasciam ao posto de saúde mais próximo de sua residência para imunização.

Dos 38 postos existentes no período da pesquisa, 10 foram sorteados, tendo sido entrevistados os pais ou responsáveis por aproximadamente 200 crianças em cada posto. Todos os responsáveis que estavam acompanhando os filhos foram convidados a participar do estudo. A taxa de recusa foi de $0,4 \%$. Os que concordaram em participar da pesquisa, responderam a um questionário contendo questões relacionadas ao nascimento das crianças, à exposição ao tabagismo passivo no domicílio e às características sócio-demográficas das famílias.

O peso e o comprimento ao nascer foram obtidos diretamente do cartão da criança; já o peso, comprimento ou estatura, no momento da pesquisa, foi medido antes de a criança ser vacinada e de ter sido realizada a entrevista. Os menores de dois anos foram pesados sem roupa, em balança pediátrica com capacidade máxima de $20 \mathrm{~kg}$ e com precisão que permitiria variação de $10 \mathrm{~g}$. Para a medida do comprimento, foi utilizada régua antropométrica de madeira, com uma peça vertical firme para a cabeça e outra móvel para os pés, estando a criança em posição supina recumbente, sobre uma superfície firme e com os joelhos esticados. As crianças com dois anos ou mais foram pesadas em balança digital, com capacidade máxima de $150 \mathrm{~kg}$ e com precisão que permitia variação de $100 \mathrm{~g}$, com o mínimo de vestuário (calcinha ou cueca), sem calçados. Para a medida da estatura foi utilizada trena metálica acoplada à haste de madeira, fixada na parede sem rodapé, com a criança em posição ortostática e braços estendidos ao longo do corpo. Um esquadro de madeira era, então, colocado acima da cabeça da criança, o qual deslizava pela haste até encostar-se na cabeça, formando um ângulo de $90^{\circ}$ entre esta e a parede. A seguir, a criança era orientada a abaixar a cabeça e sair para que se procedesse à leitura da estatura, que era registrada em local próprio no formulário da entrevista.

Os valores encontrados foram comparados com a população do National Center for Health Statistics, recomendado pela Organização Mundial da Saúde e adotado pelo Ministério da Saúde como referência para a população brasileira 11. Para a análise do estado nutricional, utilizou-se como indicador o escore $\mathrm{z}$, tendo sido avaliado o índice, estatura para a idade (E/I). Foram consideradas com baixa estatura as crianças que se encontravam com escore $\mathrm{z}$ menor que -2 desvios-padrão (DP).

Em relação à exposição domiciliar ao tabaco, as crianças foram classificadas em expostas, se morassem com pelo menos um fumante no domicílio. Investigou-se, também, a exposição da criança ao tabagismo materno, durante o período gestacional, o local da residência mais utilizado para fumar e se o fumante evitava ou não fumar na presença da criança.

O nível sócio-econômico foi avaliado pelo instrumento da Associação Brasileira de Institutos de Pesquisa de Mercado (ABIPEME) 12, tendo como base os bens existentes no domicílio (eletrodomésticos e carros), presença de empregada doméstica e escolaridade do chefe da família. O escore obtido por cada família foi tratado como variável contínua e categórica. De acordo com a pontuação obtida, as categorias variaram de A (nível mais elevado) até $\mathrm{E}$ (nível mais baixo).

A ocupação dos pais foi classificada tendo como parâmetro a Classificação Brasileira de Ocupações (CBO) 13, tendo sido agrupadas as ocupações de subgrupos relacionados, cuja média do escore $\mathrm{z}$ da estatura para idade fosse semelhante. 


\section{Análise dos dados}

Para a análise dos dados, foi utilizado o teste $\mathrm{t}$ de Student e análise de variância (ANOVA) para comparação entre as médias do escore $\mathrm{z}$ da estatura para a idade. As variáveis que se mostraram estatisticamente significantes na análise bivariada foram selecionadas para a análise multivariada. Nesta etapa, por intermédio da regressão logística, as variáveis foram incluídas em blocos, utilizando a modelagem hierárquica baseada na proposta apresentada por Victora et al. ${ }^{14}$. Foram realizadas duas análises, uma com a inclusão da variável tabagismo durante gravidez, e outra sem incluí-la na análise. Os fatores mais distais, sócio-econômicos e demográficos, foram os primeiros incluídos (nível 1), seguidos das variáveis relacionadas à criança, no nível 2 (idade e sexo) e, no nível proximal (nível 3), foi considerado o tabagismo do pai, o tabagismo da mãe e o tabagismo dos moradores. A análise que utilizou a variável tabagismo na gravidez foi incluída no nível 3, seguida das variáveis relacionadas ao tabagismo dos pais e moradores, no nível 4.

As variáveis introduzidas na análise do nível 1 foram conservadas no modelo e entraram no ajuste do nível seguinte. Em seguida, procedimento semelhante foi empregado: variáveis introduzidas no nível 2 foram mantidas para o nível 3. Variáveis proximais foram conseqüentemente, ajustadas para variáveis distais e intermediárias; porém, variáveis distais nunca foram ajustadas para variáveis proximais.

Para as análises, foram utilizados os programas Epi Info, versão 6.02 e SPSS, versão 9.0.

\section{Considerações éticas}

Anteriormente à execução do estudo, a pesquisa foi apresentada e aprovada pelo Comitê de Ética da Universidade Federal de Mato Grosso, pelo Secretário de Estado de Saúde, diretores dos distritos e gerentes dos postos de saúde em que foram desenvolvidas as entrevistas para o estudo. Antes do início da entrevista, os pais ou responsáveis eram informados sobre os objetivos da pesquisa e da possibilidade de aceitar ou rejeitar a participação de seu filho nela. Após consentimento informado, era iniciada a coleta dos dados.

\section{Resultados}

Participaram do estudo 2.037 crianças menores de 60 meses, sendo $51,4 \%$ do sexo masculino e $48,6 \%$ do sexo feminino. Dentre essas, $49,4 \%$ eram menores de 12 meses, $32,5 \%$ tinham idade entre 12 e 35 meses e 18,1\% eram maiores de 36 meses.

Do total de crianças estudadas, $37,7 \%$ eram fumantes passivas, ou seja, moravam em domicílios com pelo menos um fumante, sendo $11,2 \%$ expostas ao tabagismo da mãe, $24,3 \%$ expostas ao tabagismo do pai e $13,3 \%$ ao tabagismo dos demais moradores do domicílio.

A prevalência de crianças com baixa estatura (escore $z<-2$ ), neste estudo, foi de $4,3 \%$, sendo maior para os filhos de mães fumantes do que de mães não fumantes $(7,4 \%$ versus $3,8 \%$; risco relativo $-\mathrm{RR}=1,80$; intervalo de confiança de 95\% - IC95\%: 1,16-2,80). A média do peso e do comprimento ao nascer foi menor para as crianças cujas mães eram fumantes do que para as crianças cujas mães não eram fumantes $(3,20 \mathrm{~kg}$ versus $3,26 \mathrm{~kg}, \mathrm{p}=0,09 ; 48,4 \mathrm{~cm}$ versus $48,8 \mathrm{~cm}, \mathrm{p}=0,01)$. Do total de crianças que nasceram prematuramente (184), 38,6\% pertenciam a famílias que possuíam fumantes e $61,4 \%$ a não fumantes (RR = 0,99; IC95\%: 0,74-1,31).

Foram observadas crianças de menor estatura nos níveis sócio-econômicos mais baixos e com menor renda familiar, quando o pai não morava no domicílio, quando os pais tinham menor escolaridade e quando a criança era mais nova. Piores condições nutricionais também foram encontradas para os filhos das empregadas domésticas, dos pais que referiram ocupações na indústria e desempregados (Tabela 1).

Em relação ao hábito de fumar da mãe, verifica-se que a média do escore $\mathrm{z}$ da estatura para idade das crianças cujas mães fumaram durante o período gestacional foi estatisticamente inferior à média daquelas cujas mães não fumaram ( $p<0,0001)$. Da mesma forma, filhos de mães que relataram fumar no momento da pesquisa foram os que apresentaram a menor média do escore $\mathrm{z}$ da estatura para idade, seguidos dos filhos das ex-fumantes e não fumantes $(\mathrm{p}<0,001)$. Também foi menor a média do escore $\mathrm{z}$ da estatura para idade de crianças cujas mães relataram fumar em qualquer lugar da casa ou dentro de casa e daquelas que informaram fumar na presença dos filhos (Tabela 2).

A mesma tendência observada na média da estatura das crianças em relação ao tabagismo das mães foi verificada para os pais, ou seja, filhos de pais fumantes, que fumam em qualquer lugar da casa ou que fumam na presença dos filhos, tendem a ser mais baixos do que os demais (Tabela 3).

Em relação aos moradores fumantes do domicílio, verificou-se que a média do escore $\mathrm{z}$ da estatura para idade das crianças era tanto me- 
Média e intervalo de confiança (IC95\%) do escore z da estatura para idade, segundo a idade da criança e as variáveis sócio-demográficas das famílias. Cuiabá, Mato Grosso, Brasil; 1999.

\begin{tabular}{|c|c|c|c|}
\hline Variáveis & $\mathrm{n}$ & Média & IC95\% \\
\hline \multicolumn{4}{|l|}{ Idade da criança (meses) } \\
\hline $36 e+$ & 368 & $-0,04$ & $-0,14-0,06$ \\
\hline 12 a 35 & 663 & $-0,15$ & $-0,24-0,06$ \\
\hline \multirow[t]{2}{*}{$<12$} & 1.006 & $-0,23$ & $-0,29--0,16$ \\
\hline & \multicolumn{3}{|c|}{$p=0,02$} \\
\hline \multicolumn{4}{|l|}{ Nível sócio-econômico } \\
\hline A (mais elevado) & 78 & 0,13 & $-0,13-0,38$ \\
\hline B & 254 & 0,07 & $-0,05-0,20$ \\
\hline C & 895 & $-0,10$ & $-0,17-0,03$ \\
\hline $\mathrm{D}$ & 683 & $-0,34$ & $-0,42--0,26$ \\
\hline \multirow[t]{2}{*}{$E$ (mais baixo) } & 127 & $-0,38$ & $-0,57--0,19$ \\
\hline & \multicolumn{3}{|c|}{$p<0,0001$} \\
\hline \multicolumn{4}{|l|}{ Escolaridade da mãe } \\
\hline 2o grau completo e mais & 700 & 0,01 & $-0,07-0,09$ \\
\hline 1o grau completo & 443 & $-0,09$ & $-0,19-0,01$ \\
\hline$<$ 1o grau completo & 874 & $-0,33$ & $-0,40--0,26$ \\
\hline \multirow[t]{2}{*}{ Mães que não moram com os filhos } & 20 & $-0,99$ & $-1,90--0,12$ \\
\hline & \multicolumn{3}{|c|}{$p<0,0001$} \\
\hline \multicolumn{4}{|l|}{ Escolaridade do pai } \\
\hline 2o grau completo e mais & 667 & 0,03 & $-0,05-0,11$ \\
\hline 5a a 8a série do 1 o grau & 866 & $-0,23$ & $-0,31--0,16$ \\
\hline$<5$ a série do 1 o grau & 125 & $-0,49$ & $-0,69--0,29$ \\
\hline \multirow[t]{2}{*}{ Pais que não moram com os filhos } & 379 & $-0,28$ & $-0,39--0,17$ \\
\hline & \multicolumn{3}{|c|}{$p<0,0001$} \\
\hline \multicolumn{4}{|l|}{ Pai mora com a criança } \\
\hline Sim & 1.658 & $-0,14$ & $-0,20--0,09$ \\
\hline \multirow[t]{2}{*}{ Não } & 379 & $-0,28$ & $-0,39--0,17$ \\
\hline & \multicolumn{3}{|c|}{$p=0,03$} \\
\hline \multicolumn{4}{|l|}{ Renda per capita (salários-mínimos) } \\
\hline 2 e mais & 431 & 0,10 & $0,01-0,20$ \\
\hline $1 \mathrm{a}<2,0$ & 511 & $-0,06$ & $-0,11-0,10$ \\
\hline \multirow[t]{2}{*}{$<1,0$} & 1.095 & $-0,32$ & $-0,39--0,26$ \\
\hline & \multicolumn{3}{|c|}{$p<0,0001$} \\
\hline \multicolumn{4}{|l|}{ Ocupação da mãe* } \\
\hline Outras ocupações & 1.897 & $-0,16$ & $-0,20--0,11$ \\
\hline \multirow[t]{2}{*}{ Empregada doméstica } & 120 & $-0,24$ & $-0,44--0,03$ \\
\hline & \multicolumn{3}{|c|}{$p=0,001$} \\
\hline \multicolumn{4}{|l|}{ Ocupação do pai** } \\
\hline Outras ocupações & 1026 & $-0,06$ & $-0,12--0,01$ \\
\hline Indústria & 557 & $-0,27$ & $-0,36--0,17$ \\
\hline \multirow[t]{2}{*}{ Desempregados } & 74 & $-0,37$ & $-0,61--0,13$ \\
\hline & \multicolumn{3}{|c|}{$p=0,01$} \\
\hline
\end{tabular}

* 20 mães não moravam com os filhos no momento da entrevista;

** 379 pais não moravam com os filhos no momento da entrevista. 
Média e intervalo de confiança (IC95\%) do escore z da estatura para idade, segundo as variáveis relacionadas ao hábito de fumar da mãe da criança. Cuiabá, Mato Grosso, Brasil; 1999.

\begin{tabular}{|c|c|c|c|}
\hline Variáveis & $\mathbf{n}$ & Média & IC95\% \\
\hline \multicolumn{4}{|l|}{ Tabagismo durante a gestação } \\
\hline Não & 189 & $-0,01$ & $-0,25-0,05$ \\
\hline \multirow[t]{2}{*}{$\operatorname{Sim}$} & 210 & $-0,56$ & $-0,73--0,40$ \\
\hline & \multicolumn{3}{|c|}{$p<0,0001$} \\
\hline \multicolumn{4}{|l|}{ Hábito de fumar da mãe* } \\
\hline Não fumantes & 1.624 & $-0,12$ & $-0,17--0,07$ \\
\hline Ex-fumantes & 164 & $-0,19$ & $-0,34--0,04$ \\
\hline \multirow[t]{2}{*}{ Fumantes } & 229 & $-0,34$ & $-0,47--0,21$ \\
\hline & \multicolumn{3}{|c|}{$p=0,0009$} \\
\hline \multicolumn{4}{|l|}{ Local onde gosta de fumar } \\
\hline Não fumantes & 1.624 & $-0,12$ & $-0,17--0,07$ \\
\hline Ex-fumantes & 164 & $-0,19$ & $-0,34--0,04$ \\
\hline \multicolumn{4}{|l|}{ Fumantes } \\
\hline Fora de casa & 154 & $-0,36$ & $-0,56--0,17$ \\
\hline \multirow[t]{2}{*}{ Qualquer lugar/dentro de casa } & 75 & $-0,49$ & $-0,72--0,26$ \\
\hline & \multicolumn{3}{|c|}{$p=0,0021$} \\
\hline \multicolumn{4}{|c|}{ Evita fumar na presença da criança? } \\
\hline Não fumantes & 1.624 & $-0,12$ & $(-0,17 ;-0,07)$ \\
\hline Ex-fumantes & 164 & $-0,19$ & $(-0,34 ;-0,04)$ \\
\hline \multicolumn{4}{|l|}{ Fumantes } \\
\hline Sim & 189 & $-0,35$ & $-0,51--0,18$ \\
\hline \multirow[t]{2}{*}{ Não } & 40 & $-0,68$ & $-1,08--0,28$ \\
\hline & \multicolumn{3}{|c|}{$p=0,0006$} \\
\hline
\end{tabular}

* 20 mães não moravam com os filhos no momento da entrevista.

nor quanto maior fosse o número de moradores fumantes (Figura 1).

Na Tabela 4, observam-se os resultados da regressão linear hierarquizada. O objetivo desta análise foi verificar se o tabagismo dos pais poderia ser considerado fator de risco para a baixa estatura, mesmo após ajuste para variáveis sócio-econômicas, consideradas como variáveis de confusão para o tabagismo. No nível 1, a escolaridade da mãe e do pai, a ocupação do pai (a categoria dos pais que não moravam com os filhos no domicílio) e o nível sócio-econômico (aqui representado pela variável contínua "pontuação total") mantiveram-se associados significantemente com a estatura das crianças. No nível 2, apenas a idade da criança mostrou-se associada. Em relação aos níveis 3 e 4, verifica-se que, independentemente de ajustar-se ou não pelo tabagismo na gravidez, o tabagismo dos pais exerceu efeito próprio sobre a estatura das crianças.

\section{Discussão}

Os resultados deste estudo, à semelhança de outros 15,16,17,18, mostram que a estatura das crianças é influenciada pelo tabagismo dos pais no domicílio. Apesar de já ter sido amplamente divulgado na literatura científica que o tabagismo durante a gestação reduz o comprimento ao nascer, o tabagismo da mãe e do pai, no início da vida da criança, como foi investigado no presente estudo, também mostrou estar associado à estatura das crianças, independente do ajuste para fumo na gravidez e para outras variáveis de confusão. Optou-se pelo estudo com lactentes e pré-escolares pelo fato de essas crianças permanecerem um período longo de tempo no domicílio, em contato íntimo com os pais, sendo, portanto, mais expostas à fumaça domiciliar do tabaco.

O método de escolha para avaliar-se a exposição à fumaça domiciliar do tabaco foi um questionário respondido pelos pais, por não ser invasivo, de baixo custo e de fácil execução. Trabalhos realizados comparando valores da 
Média e intervalo de confiança (IC95\%) do escore z da estatura para idade, segundo as variáveis relacionadas ao hábito de fumar do pai da criança. Cuiabá, Mato Grosso, Brasil; 1999.

\begin{tabular}{lccc}
\hline Variáveis & $\mathbf{n}$ & Média & IC95\% \\
\hline Hábito de fumar do pai* & 1.125 & $-0,14$ & $-0,20--0,08$ \\
Não fumantes & 130 & 0,09 & $-0,07-0,26$ \\
Ex-fumantes & 403 & $-0,23$ & $-0,35--0,12$ \\
Fumantes & & $p=0,0121$ & \\
& & & \\
Local onde gosta de fumar & 1.125 & $-0,14$ & $-0,20--0,08$ \\
Não fumantes & 130 & 0,09 & $-0,07-0,26$ \\
Ex-fumantes & & & \\
Fumantes & 253 & $-0,15$ & $-0,29--0,01$ \\
$\quad$ Fora de casa & 150 & $-0,37$ & $-0,56--0,17$ \\
$\quad$ Qualquer lugar / dentro de casa & & $p=0,0061$ & \\
& & & $-0,20--0,08$ \\
Evita fumar na presença da criança? & 1.125 & $-0,14$ & $-0,07-0,26$ \\
Não fumantes & 130 & 0,09 & $-0,26--0,01$ \\
Ex-fumantes & & & $-0,84--0,29$ \\
Fumantes & 311 & $-0,14$ & $-0,56$ \\
$\quad$ Sim & 92 & $p=0,0002$ & \\
$\quad$ Não & & &
\end{tabular}

* 379 pais não moravam com os filhos no momento da entrevista.

Figura 1

Média da estatura para idade (escore z), segundo o número de fumantes do domicílio.

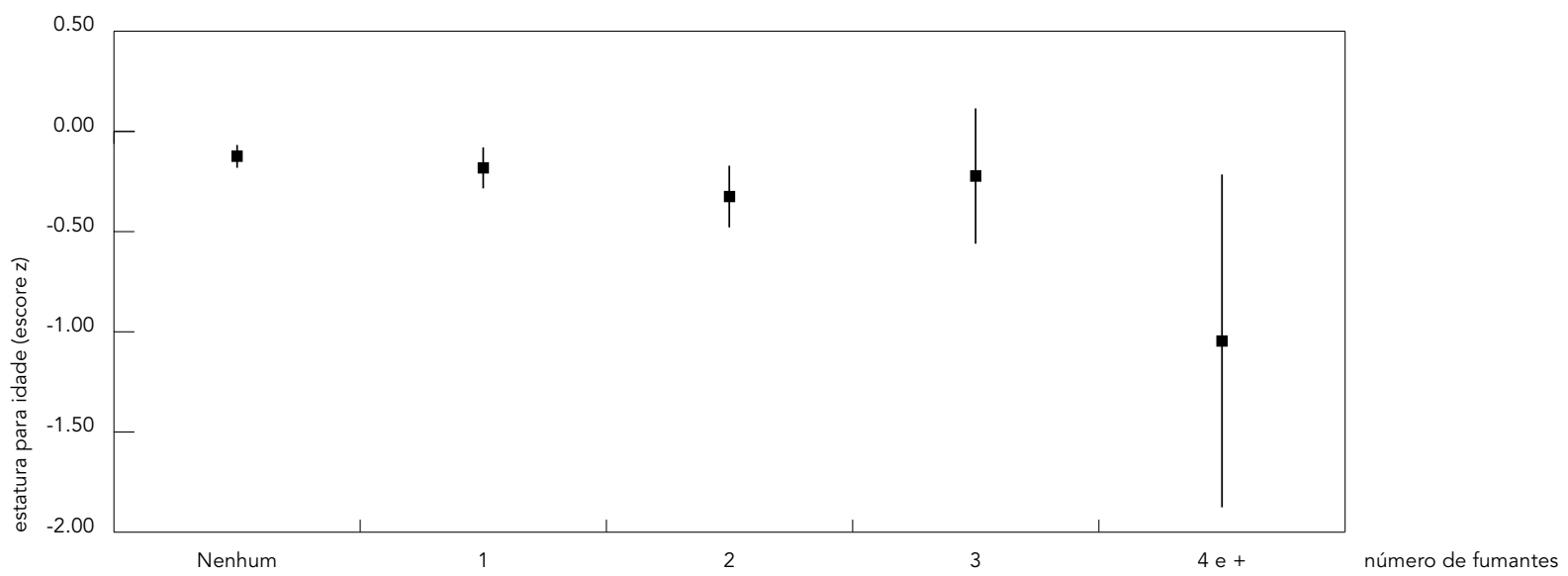


Coeficiente de regressão linear e p-valor associado entre o escore $z$ da estatura para idade (E/I)

e as variáveis sócio-demográficas das famílias e o tabagismo dos pais, segundo o nível de hierarquia.

Cuiabá, Mato Grosso, Brasil; 1999

\begin{tabular}{|c|c|c|}
\hline Níveis & Coeficiente & p-valor \\
\hline \multicolumn{3}{|l|}{ Nível 1} \\
\hline \multicolumn{3}{|l|}{ Escolaridade da mãe } \\
\hline 2o grau completo e +/ até 2o grau incompleto & 0,175 & 0,006 \\
\hline 1ㅇ grau completo e + / até 1ㅇ grau incompleto & 0,175 & 0,008 \\
\hline \multicolumn{3}{|l|}{ Escolaridade do pai } \\
\hline 2o grau completo e +/até 2 o grau incompleto & 0,859 & 0,020 \\
\hline 5a a 8a série do 1 o grau/<5a série do 1 o grau & 0,194 & 0,059 \\
\hline Sem informação */<5ạsérie do 1o grau & 0,250 & 0,032 \\
\hline \multicolumn{3}{|l|}{ Ocupação da mãe } \\
\hline $\begin{array}{l}\text { Empregada doméstica / saúde, ensino, técnicas, administração, } \\
\text { comércio, higiene, estudantes e do lar }\end{array}$ & 0,106 & 0,301 \\
\hline \multicolumn{3}{|l|}{ Ocupação do pai } \\
\hline $\begin{array}{l}\text { Indústria saúde, ensino, técnicos, administração, comércio, } \\
\text { agricultura e estudantes }\end{array}$ & $-0,061$ & 0,307 \\
\hline $\begin{array}{l}\text { Desempregados / indústria, saúde, ensino, técnicos, administração, } \\
\text { comércio, agricultura e estudantes }\end{array}$ & $-0,139$ & 0,283 \\
\hline $\begin{array}{l}\text { Sem informação*/indústria, saúde, ensino, técnicos, administração, } \\
\text { comércio, agricultura e estudantes }\end{array}$ & $-0,772$ & 0,029 \\
\hline Pontuação total (ABIPEME)** & 0,012 & 0,003 \\
\hline Salário per capita & $-0,087$ & 0,396 \\
\hline \multicolumn{3}{|l|}{ Nível 2} \\
\hline Idade da criança & 0,005 & 0,001 \\
\hline Sexo da criança & 0,072 & 0,125 \\
\hline \multicolumn{3}{|l|}{ Nível 3} \\
\hline Tabagismo na gravidez & $-0,286$ & 0,001 \\
\hline \multicolumn{3}{|l|}{ Nível 4} \\
\hline Tabagismo da mãe*** & $-0,156$ & 0,036 \\
\hline 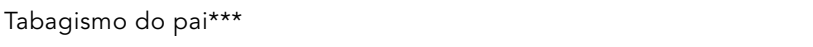 & $-0,026$ & 0,019 \\
\hline \multicolumn{3}{|l|}{ Nível 3} \\
\hline Tabagismo da mãe\# & $-0,156$ & 0,037 \\
\hline Tabagismo do pai\# & $-0,024$ & 0,031 \\
\hline
\end{tabular}

* pais que não moram com os filhos;

** classificação segundo a Associação Brasileira de Institutos de Pesquisa de Mercado 12;

*** ajustado também por tabagismo na gravidez no nível 3 .

\# não ajustado por tabagismo na gravidez. 
excreção urinária de cotinina com dados de questionário sobre o hábito de fumar dos pais mostraram-se fortemente correlacionados 19,20,21,22.

A análise permitiu controlar para tabagismo gestacional, importante variável associada ao crescimento 7,23 , revelando que, mesmo quando se ajusta para o tabagismo na gravidez, a baixa estatura associa-se ao tabagismo dos pais. Sabe-se que a estatura das crianças sofre influência de uma série de fatores. Dentre eles, os genéticos são os que irão determinar a potencialidade da estatura final do indivíduo 24 . Contudo, determinadas condições sócio-econômicas atuam de forma importante sobre o crescimento, podendo agir positiva ou negativamente sobre a estatura das crianças. A escolaridade dos pais, sobretudo a das mães, tem importância na proteção à saúde da criança, à medida que fornece meios para um melhor entendimento dos cuidados preventivos e curativos das doenças infantis e também na identificação de serviços públicos de assistência à saúde da criança 3,4 . Outra variável importante é a renda familiar, já que a mesma exerce papel fundamental na aquisição e utilização de bens e serviços essenciais como alimentação, moradia e saneamento 25 . De forma indireta, o nível de escolaridade também irá determinar as oportunidades de emprego, que irão, por sua vez, definir o nível de renda da família. Ainda que tais variáveis exerçam importante papel sobre a estatura das crianças, os resultados encontrados neste estudo demonstram que o tabagismo é uma variável que permanece significante mesmo quando se leva em conta a influência dos fatores sócio-econômicos e demográficos.

Segundo Rona et al. 15, um fator que pode explicar a associação do tabagismo passivo com o crescimento de crianças diz respeito à maior probabilidade de apresentar doenças respiratórias, que, por sua vez, poderão afetar o desenvolvimento normal dessas crianças. De fato, Cunha 26, estudando a relação entre infecção respiratória aguda e má nutrição em menores de cinco anos, verificou que a desnutrição atual e a pregressa estiveram associadas à infecção respiratória aguda, mesmo após ajuste para fatores de confusão.

Um aspecto não estudado nesta pesquisa foi a ingestão alimentar das crianças. Pode ser que os filhos de fumantes tenham tido ingestão alimentar reduzida em relação aos filhos de não fumantes, ou ainda, podem ter apresentado, em sua alimentação diária, diferente concentração de nutrientes, o que poderia de alguma forma ter influenciado seu estado nutricional.

Por outro lado, o tabaco tem em sua composição muitas substâncias que são danosas à saúde. Experimentalmente, já foi evidenciado que ratos jovens, expostos à fumaça ambiental do cigarro, tinham menor comprimento do que ratos não expostos 27 . Crianças amamentadas por mães fumantes são expostas à nicotina no leite materno, e tal exposição será tanto maior quanto menor for o tempo entre o ato de fumar da mãe e a mamada seguinte 28 . A nicotina presente no leite materno pode afetar a estatura das crianças por causa da redução da biodisponibilidade de algum nutriente essencial 18 . Alguns pesquisadores 29,30 relatam que a introdução da alimentação artificial precoce e o menor tempo de aleitamento das crianças expostas ao tabagismo passivo podem ser os responsáveis pela redução das medidas antropométricas dessas crianças, fato que não foi comprovado no presente estudo, uma vez que não houve diferença significante entre o tempo de aleitamento de crianças expostas e não expostas.

Todavia, pode ter havido, neste estudo, problemas na classificação das crianças em relação à exposição ao tabagismo. Erros de precisão na descrição do hábito de fumar (início e tempo de duração) e erros advindos da utilização da informação mais recente sobre o fumo podem ter ocorrido. Estudo recente 10, utilizando os mesmos dados, mostrou menores prevalências de tabagismo durante o primeiro ano de vida das crianças, o que pode indicar uma alteração do hábito de fumar dos moradores quando a criança é pequena. A informação obtida transversalmente, no momento da entrevista, foi utilizada como tendo sido constante durante toda a vida da criança, vale dizer, uma informação transversal e instantânea foi utilizada para estimar uma informação longitudinal e dinâmica, no que pode ter gerado um erro de classificação das crianças quanto à exposição ao fumo passivo.

Conclui-se pela importância do tabagismo como um dos fatores ambientais que interferem no crescimento, sugere-se que o tabagismo seja incluído nos estudos de avaliação nutricional e antropométrica, por ser uma variável que pode, biologicamente, interferir no crescimento da criança e também porque medidas de controle precisam ser utilizadas e implementadas para reduzir sua prevalência na população, contribuindo para estilos de vida mais saudáveis para nossas crianças. 


\section{Resumo}

O tabagismo durante a gestação é um dos responsáveis pelo menor peso e comprimento ao nascer. No entanto, a exposição à fumaça do tabaco, no período pós-natal, não tem sido explorada nos estudos de crescimento. Sabe-se que a prevalência do tabagismo é alta no nível sócio-econômico mais baixo e que a estatura de crianças está também associada com variáveis sócio-econômicas. O objetivo deste estudo foi verificar o efeito das variáveis sócio-econômicas e da exposição à fumaça do tabaco sobre o crescimento. Foram medidos e pesados os menores de cinco anos atendidos nos postos de saúde, para imunização $(n=2.037)$. Os pais responderam a um questionário sobre o tabagismo no domicílio e características sócio-demográficas das famílias. A prevalência da baixa estatura foi 4,3\%. Verificou-se na análise bivariada associação negativa entre a estatura e o tabagismo gestacional, e associações positivas com nível sócio-econômico, renda familiar e escolaridade dos pais. A análise de regressão linear hierarquizada mostrou que o tabagismo dos pais permaneceu associado com a baixa estatura mesmo após ajuste para tabagismo durante a gravidez e para variáveis sócio-demográficas.

Criança; Estatura; Poluição por Fumaça de Tabaco

\section{Colaboradores}

R. M. V. Gonçalves-Silva colaborou em todas as etapas do trabalho. J. G. Valente participou desde o planejamento até a fase final do trabalho. M. G. F. Lemos-Santos contribuiu com a coleta de dados. R. Sichieri participou da análise dos dados e redação do trabalho.

\section{Referências}

1. Gallo P, Amigo H, Cláudio L. Fatores de risco ao retardo de crescimento estatural em crianças de baixo nível econômico e social de São Paulo, Brasil. Arch Latinoam Nutr 2000; 50:121-5.

2. Engstrom EM, Anjos LA. Déficit estatural nas crianças brasileiras: relação com condições sócio-ambientais e estado nutricional materno. Cad Saúde Pública 1999; 15:559-67.

3. Monteiro CA, Zuñiga HPP, Benício MHD, Szarfarc SC. Estudo das condições de saúde das crianças do Município de São Paulo, SP (Brasil), 1984-1985. I - Aspectos metodológicos, características sócioeconômicas e ambiente físico. Rev Saúde Pública 1986; 20:435-45.

4. Guimarães LV, Latorre MRDO, Barros MBA. Fatores de risco para a ocorrência de déficit estatural em pré-escolares. Cad Saúde Pública 1999; 15: 605-15.

5. Kukla L, Hruba D, Tyrlik M. Smoking and damages of reproduction: evidence of ELSPAC. Cent Eur J Public Health 2001; 9:59-63.

6. Shankaran S, Das A, Bauer CR, Bada HS, Lester B, Wright LL, et al. Association between patterns of maternal substance use and infant birth weight, length, and head circumference. Pediatrics 2004; 114:226-34.

7. Hanke W, Sobala W, Kalinka J. Environmental tobacco smoke exposure among pregnant women: impact on fetal biometry at 20-24 weeks of gestation and newborn child's birth weight. Int Arch Occup Environ Health 2004; 77:47-52.

8. Eskenazi B, Bergmann JJ. Passive and active smoking during pregnancy, as measured by serum cotinine, and postnatal smoke exposure. I. Effects on physical growth at age 5 years. Am J Epidemiol 1995; 142 (9 Suppl):S10-8.

9. Chinn S, Rona RJ. Quantifying health aspects of passive smoking in British children aged 5-11 years. J Epidemiol Community Health 1991; 45:188-94.

10. Gonçalves-Silva RMV, Valente JG, Lemos-Santos MGF, Sichieri R. Tabagismo domiciliar em famílias com crianças menores de 5 anos no Brasil. Rev Panam Salud Publica 2005; 17:163-9.

11. Instituto Nacional de Alimentação e Nutrição. Pesquisa nacional sobre saúde e nutrição: perfil de crescimento da população brasileira de 0 a 25 anos. Brasília: Ministério da Saúde, 1990.

12. Associação Brasileira dos Institutos de Pesquisa de Mercado. Proposição para um novo critério de classificação sócio-econômica. São Paulo: Associação Brasileira dos Institutos de Pesquisa de Mercado; 1978.

13. Secretaria de Políticas de Emprego e Salário, Ministério do Trabalho e Emprego. Tábua de conversão CBO - 94. Brasília: Secretaria de Políticas de Emprego e Salário, Ministério do Trabalho e Emprego; 1996.

14. Victora CG, Huttly SR, Fuchs SC, Olinto MTA. The role of conceptual frameworks in epidemiological analysis: a hierarchical approach. Int J Epidemiol 1997; 26:224-7.

15. Rona RJ, Chinn S, Florey CD. Exposure to cigarette smoking and children's growth. Int J Epidemiol 1985; 14:402-9. 
16. Leger J, Limoni C, Czernichow P. Prediction of the outcome of growth at 2 years of age in neonates with intra-uterine growth retardation. Early Hum Dev 1997; 48:211-23.

17. Salazar G, Garcia C, Berlanga R, Ahumada M, Seron-Ferre M, Vio F. Efecto del tabaquismo materno en el crecimiento de sus hijos. Rev Med Chil 1998; 126:1059-64.

18. Berlanga MR, Salazar G, Garcia C, Hernandez J. Maternal smoking effects on infant growth. Food Nutr Bull 2002; 23 Suppl 3:142-5.

19. Slattery ML, Hunt SC, French TK, Ford MH, Williams RR. Validity of cigarette smoking habits in three epidemiologic studies in Utah. Prev Med. 1989; 18:11-9.

20. Henschen M, Frischer T, Pracht T, Spiekerkötter E, Karmaus W, Meinert R, et al. The internal dose of passive smoking at home depends on the size of the dwelling. Environ Res 1997; 72:65-71.

21. Gilliland FD, Li YF, Peters JM. Effects of maternal smoking during pregnancy and environmental tobacco smoke on asthma and wheezing in children. Am J Respir Crit Care Med 2001; 163:429-36.

22. Carvalho LMT, Pereira EDB. Morbidade respiratória em crianças fumantes passivas. J Pneumologia 2002; 28:8-14.

23. Jaakkola JJ, Jaakkola N, Zahlsen K. Fetal growth and length of gestation in relation to prenatal exposure to environmental tobacco smoke assessed by hair nicotine concentration. Environ Health Perspect 2001; 109:557-61.
24. Rona RJ, Chinn S. Genetic and environmental influences on growth. J Med Screen 1995; 2:133-9.

25. Cesar JA, Horta BL, Gomes G, Shehadeh I, Chitolina J, Rangel L, et al. Utilização de serviços de saúde por menores de cinco anos no extremo Sul do Brasil. Cad Saúde Pública 2002; 18:299-305.

26. Cunha AL. Relationship between acute respiratory infection and malnutrition in children under 5 years of age. Acta Paediatr 2000; 89:608-9.

27. Gonçalves-Silva RMV, Lemos-Santos MGF, Botelho C. Influência do tabagismo no ganho ponderal, crescimento corporal, consumo alimentar e hídrico de ratos. J Pneumologia 1997; 23:124-30.

28. Dahlstrom A, Ebersjo C, Lundell B. Nicotine exposure in breastfed infants. Acta Paediatr 2004; 93:810-6.

29. Fried PA, Watkinson B, Gray R. Growth from birth to early adolescence in offspring prenatally exposed to cigarettes and marijuana. Neurotoxicol Teratol 1999; 2:513-25.

30. Nafstad P, Jaakkola JJ, Hagen JA, Pedersen BS, Ovigstad E, Botten G, et al. Weight gain during the first year of life in relation to maternal smoking and breast feeding in Norway. J Epidemiol Community Health 1999; 51:261-5.

Recebido em 05/Out/2004

Versão final reapresentada em 22/Mar/2005

Aprovado em 18/Abr/2005 